\title{
Conceptual Models for Fracture Characterization at Reykjanes Geothermal Field
}

\author{
Lilja Magnusdottir $^{1}$ Magnus Thor Jonsson ${ }^{2}$ \\ ${ }^{1}$ Ind. Eng, - Mech. Eng, and Computer Science, University of Iceland, Iceland, li l jamag@ hi . is \\ ${ }^{2}$ Ind. Eng, - Mech. Eng, and Computer Science, University of Iceland, Iceland, magnusj@hi . is
}

\begin{abstract}
This paper describes conceptual models for an inverse analysis to investigate fracture topology at Reykjanes geothermal reservoir using electrical resistivity. The resistivity distribution of a field can be estimated by measuring potential differences between various points while injecting an electric current into the ground, and resistivity data can be used to infer fracture properties due to the large contrast in resistivity between water and rock. Furthermore, injecting water into the reservoir with higher resistivity than the conductive brine at Reykjanes would cause a time-dependent change in the resistivity of the fractures as the fluid flows through the fracture network. Thus, modeling the time history of the potential difference between two points (e.g. an injector and a producer) as water is injected into the reservoir and comparing simulated results to actual observation that depend on the fracture network in the field, could help estimate where fractures are located and the characterize their distribution.
\end{abstract}

Keywords: Fracture characterization, Reykjanes, IDDP, electrical resistivity, ERT

\section{Introduction}

Reykjanes geothermal field in Iceland is located about $50 \mathrm{~km}$ southwest of Reykjavík on the Reykjanes peninsula. At Reykjanes geothermal field, an existing well (RN-15) has been deepened down to a $4.7 \mathrm{~km}$ depth as a part of the Iceland Deep Drilling Project (IDDP) (Fridleifsson et al., 2017). Supercritical fluids can exist near heat sources in magmatic reservoirs and at Reykjanes the temperature is expected to reach up to $500^{\circ} \mathrm{C}$ in the deepened well (IDDP-2). At temperatures and pressures above the critical point $\left(374^{\circ} \mathrm{C}\right.$ and 22.064 $\mathrm{MPa}$ ), the fluid has multiple power-producing potentials compared to fluid produced in conventional geothermal power plants. The enthalpy is significantly higher at such high temperatures and pressures, and the rates of mass transfer is greatly enhanced due to the increased ratios of buoyancy forces to viscous forces in the supercritical state. Thus, supercritical fluid is desired for optimized power productions since more energy could be produced from a single well extracting supercritical fluids compared to a conventional geothermal well.
For optimizing power production at Reykjanes, it is crucial to study the behavior of the fluid and heat transfer at the deep roots of the system. Connectivity of fractures is essential to ensure adequate supply of geothermal fluids and efficient thermal operation of the wells in the reservoir. The knowledge of the configuration of fractures and the fluid-flow patterns is extremely valuable for designing the recovery strategy appropriately, optimizing the placing of injection or production wells, and increasing the overall efficiency of the power production.

In this paper, a conceptual model of Reykjanes is described for estimating fracture connectivity by measuring time-lapse electric potential data while injecting a fluid into the reservoir with different conductivity than the geothermal fluid. If the injected fluid is less conductive than the geothermal fluid, the electric potential will increase as the injected fluid fills fractures from the injector towards the producers. Therefore, the time-lapse electric potential data are related to the connectivity of the fracture network.

A variety of approaches have been attempted to use time-dependent geophysical data that can indirectly measure time-varying hydrologic parameters (DayLewis et al., 2003; Lambot et al., 2004). Electrical resistivity has been shown to be sensitive to changes in fluid conductivity and water content in reservoirs (e.g., Binley et al., 2002; Yeh et al., 2002), and the concentration of a conductive tracer can be mapped from field measurements of resistance using cross-well Electrical Resistivity Tomography (ERT) (Singha and Gorelick, 2006). A number of studies have demonstrated the potential of ERT for monitoring tracer migration in soil, (Binley et al., 1996; Köstel et al., 2008; Olsen et al., 1999; Slater et al., 2000), and in shallow aquifers (Binley et al., 2002; Cassiani et al., 2006; Oldenborger et al., 2007; Singha and Gorelick, 2005; Singha et al., 2007). In these studies, usually many electrodes were used to obtain the resistivity distribution for the whole field under study at each time step and then this resistivity distribution was compared to the distribution without any tracer to observe resistivity changes in each block visually. Using this 
approach for a whole reservoir would require a massive parameter space and likely not be solvable, except at very low resolution. However, in the method considered in this study, the potential difference between the wells which corresponds to the changes in resistivity is measured and plotted as a function of time while the injected water flows through the fracture network. Then, this time-lapse electric potential is used in an inverse analysis to estimate the connectivity between points (e.g. wells) in the reservoir.

The proposed plan includes first developing and verifying an iTOUGH2 model of Reykjanes geothermal reservoir. The model's depth has to be at least $5 \mathrm{~km}$ due to well RN-15 that has been deepened down to a $4.7 \mathrm{~km}$ depth. The plan involves using the supercritical module in iTOUGH2, EOS1SC, which allows for simulating the extreme temperature and pressure conditions at the roots of geothermal reservoirs (Magnusdottir and Finsterle, 2015). That way, the heat source can be included in the model. Then, the model is calibrated with an inverse analysis using production data from the field.

Next, an inverse analysis is proposed for estimating the fracture connectivity in the reservoir using timelapse electric potential data as water with lower conductivity than the geothermal fluid is injected into the reservoir. The inverse analysis first requires a fracture model that can be created using a Discrete Fracture Network (DFN) based on the $>5 \mathrm{~km}$ deep iTOUGH2 model of Reykjanes. Then, the flow through the reservoir of injected water needs to be simulated using iTOUGH2. In addition, the analogy between Ohm's law that describes electrical flow and Darcy's law describing fluid flow makes it possible to use iTOUGH2 to calculate the electric fields. Finally, the inverse analysis requires measuring the electric field in the reservoir for comparison to the simulated electric fields in order to estimate the fracture connectivity.

\section{Numerical Model of Reykjanes}

In geothermal modeling, the roots of the geothermal systems are normally avoided but in order to accurately predict the thermal behavior when wells are drilled close to magmatic intrusions, it is necessary to incorporate the heat sources into the modeling scheme. Thus, it is recommended to use the supercritical module in iTOUGH2, EOS1SC, for creating a $>5 \mathrm{~km}$ deep numerical model of Reykjanes including the heat source at the bottom. Figure 1 shows an example of a grid in $\mathrm{X}$ and $\mathrm{Y}$ direction for a model of Reykjanes around well $\mathrm{RN}-15$ with a finer grid where the wells are located. This grid can then be copied for the multiple layers in $\mathrm{Z}$ direction that represents the depth of the model. The model parameters are chosen to generally fit the measured data and other knowledge regarding the reservoir. Lower permeability is defined for the elements at the boundaries and the inverse analysis focuses on the permeability distribution of the smaller

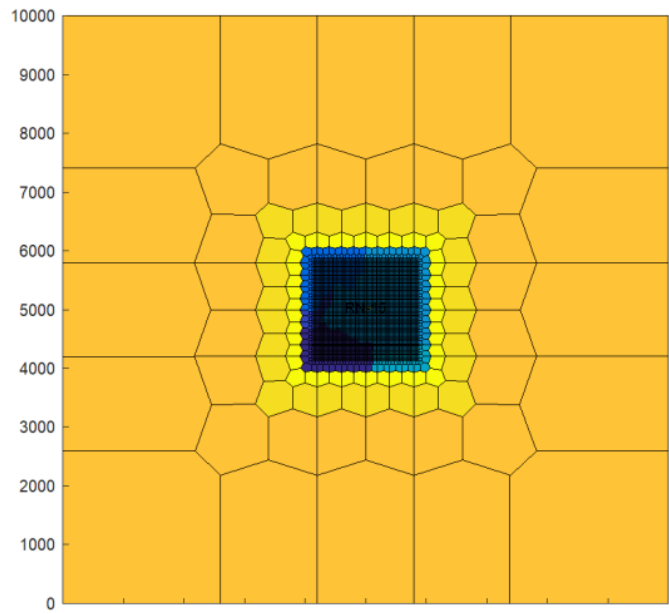

Figure 1. An example of a grid in $\mathrm{X}$ and $\mathrm{Y}$ direction for a model of Reykjanes. Color represents rock properties.

element net in the middle of the grid in Figure 1. The reservoir model is first simulated without any production until it reaches a steady-state. Then, production is simulated. The simulated pressure and temperature in the reservoir before and during the production is used to calibrate the model by comparing the simulated data to actual observations in the field. The input parameters for the inverse analysis are the permeability distribution in the field as well as the temperature of the heat source at the bottom and the output parameters are the production data and the measured pressure and temperature before production started. A possible solution for the permeability and temperature in the reservoir has been established once there is a good fit between the simulated output parameters and the observations in the field. Figure 2 shows an example of a fit between simulated and observed pressure and temperature for well RN-15 in Reykjanes at steady-state. The red data points are the measured data and the blue data points are the simulated data. For this example, the simulated data already fit relatively well to measured data. The next step would be to simulate the production and perform an inverse analysis to get a better fit.

\section{Inverse Analysis for Fracture Characterization}

In previous section, we discussed how in this study a numerical reservoir model would be first created based on an observation of the reservoir and measurements taken in the field. Then, in order to take full advantage of the data available for model construction, inverse analysis is used to calibrate the model. In inverse modeling, the results of actual observations such as measurements of pressure and temperature in the field or electrical resistivity measurements are used to infer the values of the parameters characterizing the system. In previous section we described how an inverse analysis can be used to estimate the permeability and the 

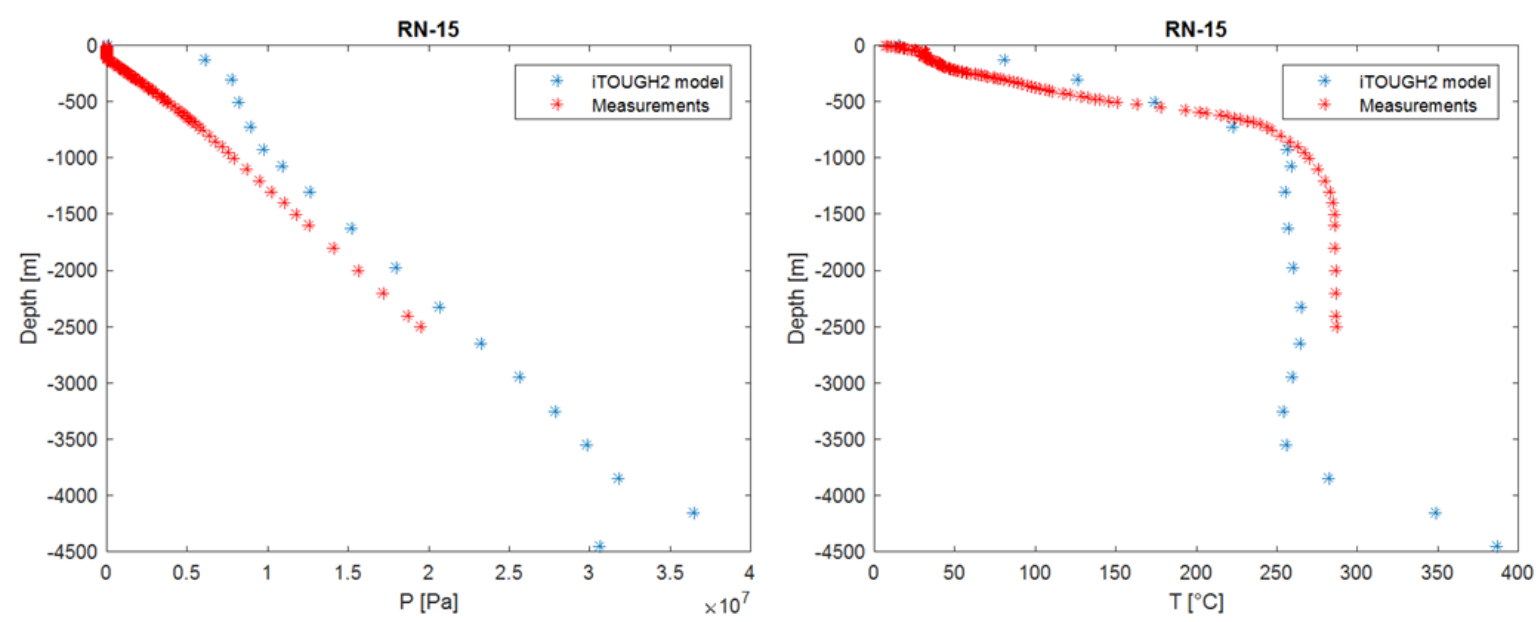

Figure 2. The measured pressure and temperature distribution at well RN-15 (now IDDP-2) before any production, compared to the steadystate pressure and temperature distribution in an iTOUGH2 model of Reykjanes.

temperature of a heat source in the reservoir for the model of Reykjanes using pressure and temperature measurements. Furthermore, this section will explain how inverse analysis can be used to characterize the fracture connectivity in the reservoir using electrical resistivity measurements. The output parameters are the potential differences between wells as a function of time and the input parameter is the character of the corresponding fracture network. The objective function measures the difference between the model calculation, which in this case is the calculated voltage difference between wells, and the corresponding observed data measured in the reservoir.

The inverse analysis is further illustrated in Figure 3. First, a fracture model is developed based on the existing iTOUGH2 model and observations in the field. The plan includes using a Discrete Fracture Network (DFN) model introduced by Karimi-Fard et al. (2003) so realistic fracture patterns can be modeled. DFN models represent fracture-dominated flow paths in geothermal reservoirs more accurately since the fractures are treated discretely instead of being defined by high permeability values in course-scale grid blocks, as done in the iTOUGH2 model previously described.

Once a conceptual fracture model of the reservoir has been constructed, a flow simulation is performed using iTOUGH2 to study how injected water flows through the fracture network of the reservoir. It is important that the conductivity of the water injected is lower than the conductivity of the fluid in the reservoir so that a change in the electric potential is produced between the injection well and the production well. Then, the electric potential field is solved for each time step as the injected water is flowing through the reservoir and the electric potential difference between the wells is recorded. iTOUGH2 can also be used to calculate the electric fields as explained in the next section. Then, this time-history of the simulated electric potential difference is compared to the true measured potential difference in the reservoir in the inverse analysis. The fracture characteristics of the fracture model are changed until a good match has been established between the simulated potential difference and the potential difference observed in the reservoir during measurements. Inverse analysis is a powerful tool for validating the model and predicting the fracture connectivity in the reservoir as explained by Magnusdottir and Horne (2013).

\section{Electric Field Simulated Using iTOUGH2}

The potential distribution in steady-state porous flow is exactly the same as the potential distribution in an electric conducting medium due to the analogy between Darcy's law and Ohm's law, as formulated by Muskat (1932). Therefore, flow simulator iTOUGH2 can be used to calculate both the distribution of water injected to change the resistivity of the fractures in the reservoir and to calculate the electric field at each time step. The same grid can then be used for both electric and fluid flow models, making the simulation more efficient than if separate models were used.

Darcy's law is an empirical relationship similar to Ohm's law,

$$
J=-\sigma \nabla \varphi
$$

where $J$ is current density $\left[\mathrm{A} / \mathrm{m}^{2}\right], \sigma$ is the conductivity of the medium $[\Omega \mathrm{m}]$ and $\varphi$ is the electric potential $[\mathrm{V}]$, but instead of describing electrical flow Darcy's law describes fluid flow through a porous medium,

$$
q=-\frac{k}{\mu} \nabla p
$$

where $q$ is the flow rate $[\mathrm{m} / \mathrm{s}], k$ is permeability $\left[\mathrm{m}^{2}\right]$, $\mu$ is viscosity of the fluid $[\mathrm{kg} / \mathrm{ms}]$ and $p$ is pressure $[\mathrm{Pa}]$. Table 1 presents the correspondence between the variables and relations of water flow (Darcy's law) and electric current flow (Ohm's law). 

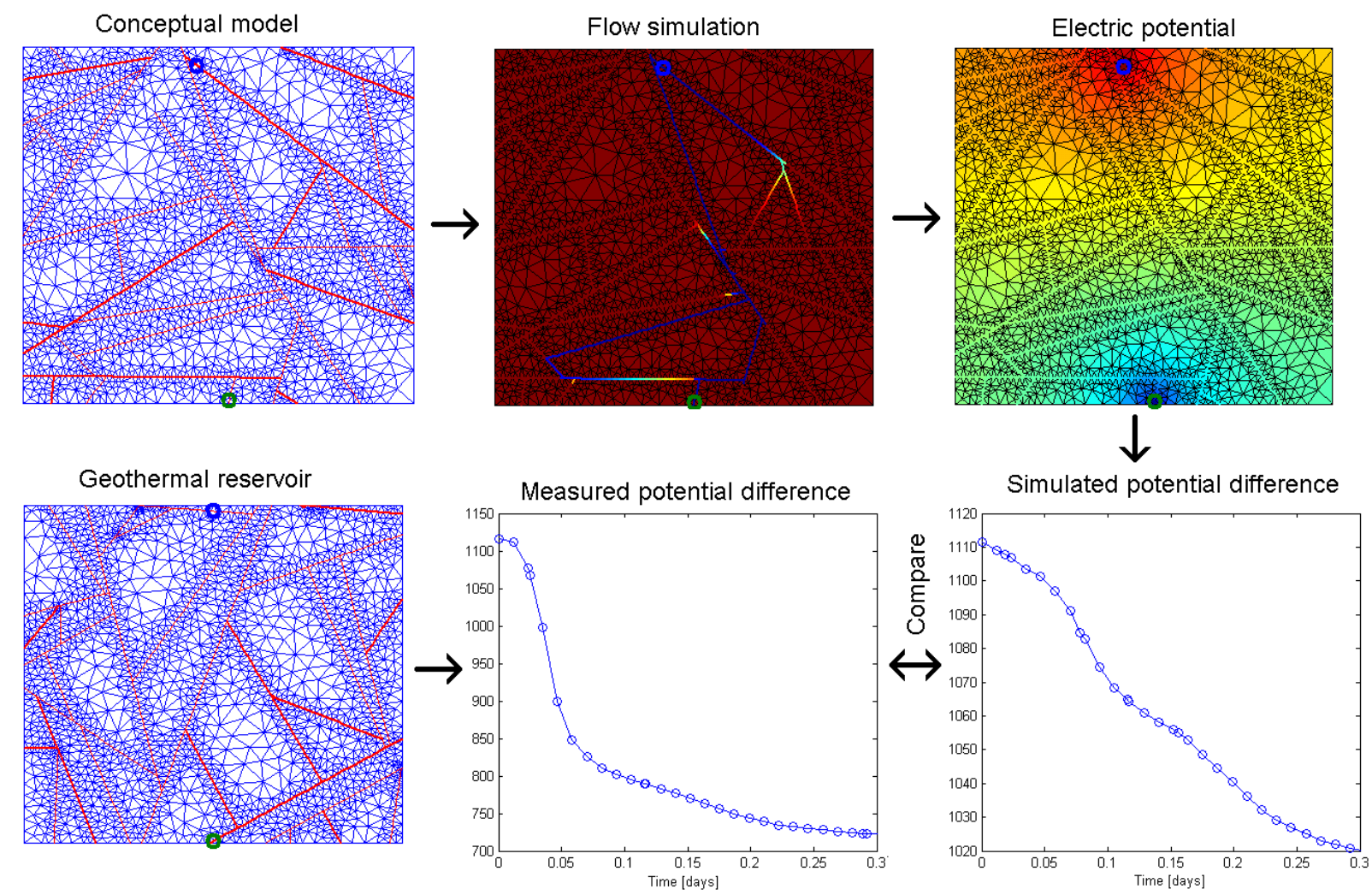

Figure 3. Schematic illustration of the inverse analysis proposed in this paper.

Table 1. Correspondence between electric current flow and water flow.

\begin{tabular}{|l|l|l|}
\hline & Darcy's law: & Ohm's law: \\
\hline Flux of: & Water $q[\mathrm{~m} / \mathrm{s}]$ & Charge $J\left[\mathrm{~A} / \mathrm{m}^{3}\right]$ \\
\hline Potential: & Pressure $p[\mathrm{~Pa}]$ & Voltage $\varphi[\mathrm{V}]$ \\
\hline $\begin{array}{l}\text { Medium } \\
\text { property: }\end{array}$ & $\begin{array}{l}\text { Hydraulic } \\
\text { conductivity }\end{array}$ & $\begin{array}{l}\text { Electrical } \\
\text { conductivity } \\
\end{array}$ \\
$\frac{k}{\mu}[1 / \Omega \mathrm{m}]$
\end{tabular}

The pressure results from iTOUGH2 correspond to the electric voltage, the current density to the flow of water and the electrical conductivity corresponds to the hydraulic conductivity, i.e.

$$
\sigma=\frac{k}{\mu}
$$

However, it must be taken into account that viscosity depends on pressure while conductivity of a reservoir does not depend on the electric voltage used. In order to take the pressure dependence into account, the EOS9 module in iTOUGH 2 can be used. EOS 9 considers flow of a single aqueous phase consisting of a single water component. The conditions are assumed to be isothermal so only a single water mass balance equation is solved for each grid block and the thermal properties of water can be overwritten. Therefore, liquid viscosity, density and compressibility can be defined constant and reference pressure and temperature can be overwritten in iTOUGH2, making the imitation of electric flow possible.

\section{Measurements}

The final crucial part of the inverse analysis is observations in the field. Measurements of the electric current between the wells as the injected water flows through the reservoir gives information about the timelapse resistivity in the field. The measurments are necessary for estimating the characteristics of the fracture model.

Well RN-15 in Reykjanes seems ideal for this experiment because once it was deepened during the Iceland Deep Drilling Project (IDDP), it was disconnected from the pipelines that go towards the powerplant and connect the other wells together. Thus, during experiments, the current will not travel from well $\mathrm{RN}-15$ to other wells via these pipelines and will instead travel down the electrode (placed on the surface or inside the well, or the steel casings themselves are used as electrodes) into the ground. For measurements, it's important that significant current is going into the ground from one measurement point to another and not through the pipelines so the measured data will give information about the fracture connectivity in the ground. 


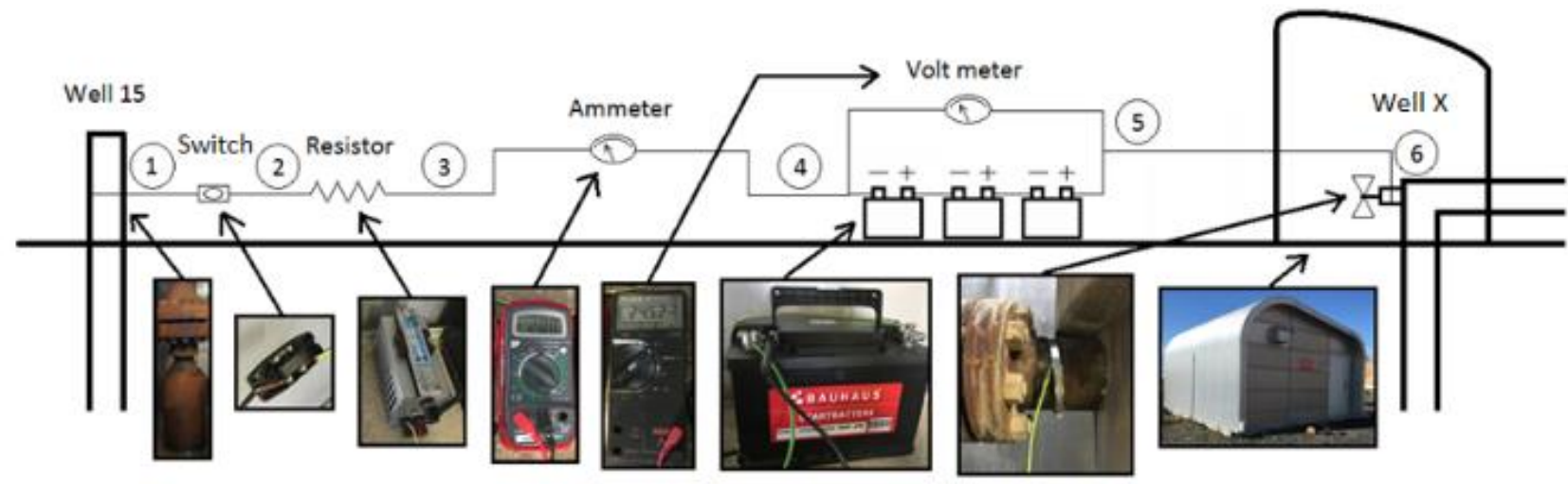

Figure 4. An example of a measurement setup at Reykjanes.

Figure 4 shows an example of a setup for measuring the resistivity between well RN-15 and another well as water is being injected into the reservoir. The setup can be constructed as follows. For using the steel casings as electrodes, an electric wire can be attached to the production casing of well $\mathrm{RN}-15$ with a hose clamp. It is important to file down part of the casing to assure a good connection to the wire. Next, a switch is attached to the wire from well $\mathrm{RN}-15$ to ease the process of connecting and disconnecting the electric circuit. Then, a resistor is attached to the circuit to control the amount of current flowing through the circuit. An ammeter is added to measure the current flowing through the circuit and a volt meter to measure the electric potential difference over one and up to three batteries. The batteries are $12 \mathrm{~V} 72 \mathrm{Ah}$ batteries that should be connected in series when more than one battery is used. Then, the electric wire coming from well RN-15 connects to the negative terminal and the positive terminal connects to a casing (or a valve connected to the casing as shown in Figure 4) using a hose clamp on the other well being tested.

If the switch would be pressed so the circuit is closed, electric current flows from the positive terminal of the battery, down the production casing of well A in Figure 4 , through the reservoir, up the production casing of well $\mathrm{RN}-15$ and through the circuit to the negative terminal of the battery. Thus, the measured current and voltage will give information about the resistivity of the path the current travels through the reservoir. As fluid with higher resistivity than the fluid in the reservoir is injected, the measured resistivity between the wells will increase. This time-lapse resistivity can then be used in the inverse analysis as explained in previous section, to give information about the fracture connectivity in the reservoir.

\section{Conclusions}

This paper describes a conceptual model for estimating fracture connectivity in Reykjanes geothermal reservoir where a well has been drilled to a depth of $4.7 \mathrm{~km}$. The proposed method includes modeling changes in resistivity as injected water with lower conductivity than the fluid in the reservoir flows through the fracture network. Then, measurements of the time-lapse electric data are used in an inverse analysis to estimate the connectivity of the fracture data.

There are three conceptual models discussed in this paper; an over $5 \mathrm{~km}$ deep numerical model of Reykjanes where the permeability distribution and the temperature of the heat source are estimated using production data from the field, a fracture model where a discrete-fracture method is used to model realistic fracture networks, and a model for simulating the electric fields in the reservoir. All three models are created using iTOUGH2. Finally, the necessary measurements are discussed for using the models in an inverse analysis to estimate the fracture connectivity in the reservoir. The knowledge of the heat transfer and fluid-flow patterns in the reservoir is extremely valuable for efficient management of the system and optimal power production.

\section{Acknowledgements}

This research was supported by The Icelandic Research Fund, project number 163514-052.

\section{References}

Binley A., Henry-Poulter S., Shaw B. (1996) Examination of Solute Transport in an Undisturbed Soil Column Using Electrical Resistance Tomography. Water Resour. Res. 32(4):763-769. Doi:10.1029/95WR02995.

Binley A., Giorgio C., Middleton R., Winship P. (2002) Vadose zone flow model parameterisation using crossborehole radar and resistivity imaging. Journal of Hydrology 267:147-159. Doi:10.1016/S00221694(02)00146-4.

Cassiania G., Brunoa V., Villaa A., Fusia N. Binley A. (2006) A saline trace test monitored via time-lapse surface electrical resistivity tomography. Journal of Applied Geophysics 59:244-259. doi:10.1016/ j.jappgeo.2005.10.007.

Day-Lewis F. D., Lane Jr. J. W, Harris J. M, Gorelick S. M. (2003) Time-lapse imaging of saline-tracer transport in 
fractured rock using difference-attenuation radar tomography. Water Resour. Res. 39:1290. Doi:10.1029/2002WR001722,10.

G. O. Fridleifsson, W. Elders, R. Zierenberg, A. Stefansson, O. Sigurdsson, Th. Gislason, T. B. Weisenberger, B. S. Hardarson, K. G. Mesfin (2017) ICDP supported coring in IDDP-2 at Reykjanes - the DEEPEGS demonstrator in Iceland - Supercritical conditions reached below $4.6 \mathrm{~km}$ depth. 19th EGU General Assembly, proceedings from the conference held 23-28 April, 2017 in Vienna, Austria., p.14147.

Karimi-Fard M., Durlofsky L. J., Aziz K. (2003) An Efficient Discrete Fracture Model Applicable for General Purpose Reservoir Simulators. SPE 79699, SPE Reservoir Simulation Symposium, Houston, TX.

Koestel J., Kemna A., Javaux M., Binley A., Vereecken H. (2008) Quantitative imaging of solute transport in an unsaturated and undisturbed soil monolith with 3-D ERT and TDR. Water Resour. Res. 44. Doi:10.1029/ 2007WR006755.

Lambota S., Antoinea M., Boschb I. van den, Slobc E. C., Vancloostera M. (2004) Electromagnetic Inversion of GPR Signals and Subsequent Hydrodynamic Inversion to Estimate Effective Vadose Zone Hydraulic Properties. Vadose Zone Journal 3:1072:1081. Doi:10.2113/3.4.1072.

Magnusdottir, L., Finsterle, S. (2015) An iTOUGH2 equationof-state module for modeling supercritical con in geothermal reservoirs, Geothermics, 57, 8-17. doi:10.1016/ j.geothermics.2015.05.003.

Magnusdottir, L., Horne, R. (2013) Inversion of time-lapse electrical resistivity data to estimate fracture connectivity. Mathematical Geosciences, 47, 85-104. Doi:10.1007/s11004-013-9515-9
Muskat M. (1932) Potential Distributions in Large Cylindrical Disks with Partially Penetrating Electrodes. Physics 2:329364. Doi:10.1063/1.1745061.

Oldenborger G. A., Knoll M. D., Routh P. S., LaBrecque D. J. (2007) Time-lapse ERT monitoring of an injection/withdrawal experiment in a shallow unconfined aquifer. Geophysics 72:F177-F187. Doi:10.1190/ 1.2734365 .

Olsen P. A., Binley, A., Henry-Poulter, S. and Tych, W. (1999) Characterizing solute transport in undisturbed soil cores using electrical and X-ray tomographic methods. Hydrol. Process. 13:211-221. Doi:10.1002/(SICI)10991085(19990215)13:2<211::AID-HYP707>3.0.CO;2-P.

Singha K., Day-Lewis F. D., Moysey S. (2007) Accounting for tomographic resolution in estimating hydrologic properties from geophysical data. Geophysical Monograph Series 171:227-241.

Singha K., Gorelick S. M. (2005) Saline Tracer Visualized with Three-dimensional Electrical Resistivity Tomography: Field-scale Spatial Moment Analysis. Water Resour. Res. 41. Doi:10.1029/2004WR003460.

Singha K., Gorelick S. M. (2006) Hydrogeophysical tracking of three-dimensional tracer migration: The concept and application of apparent petrophysical relations. Water Resour. Res. 42. Doi:10.1029/2005WR004568.

Slater L., Binley A. M., Daily W, Johnson R. (2000) Crosshole Electrical Imaging of a Controlled Saline Tracer Injection. Journal of Applied Geophysics 44:85-102. Doi:10.1016/S0926-9851(00)00002-1.

Yeh T.-C. J., Liu S., Glass R. J., Baker K., Brainard J. R., Alumbaugh D. L., LaBrecque D. (2002) A geostatistically based inverse model for electrical resistivity surveys and its applications to vadose zone hydrology. Water Resour. Res. 38(12). Doi:10.1029/2001WR001204. 